Article

\title{
Implementation of a Blood Cold Chain System Using Blockchain Technology
}

\author{
Seungeun Kim ${ }^{1}$, Joohyung Kim ${ }^{2}$ and Dongsoo Kim ${ }^{2, *}$ \\ 1 Department of IT Distribution and Logistics, Soongsil University, 369 Sangdo-Ro, Dongjak-Gu, \\ Seoul 06978, Korea; sungkim@soongsil.ac.kr \\ 2 Department of Industrial and Information Systems Engineering, Soongsil University, 369 Sangdo-Ro, \\ Dongjak-Gu, Seoul 06978, Korea; kjh512@ssu.ac.kr \\ * Correspondence: dskim@ssu.ac.kr
}

Received: 10 April 2020; Accepted: 8 May 2020; Published: 11 May 2020

\begin{abstract}
As the population structure changes due to lower fertility rates and rapid aging, the blood supply available for blood transfusion decreases and demand increases. In most countries, blood management information systems, led by national institutions, operate centrally. However, existing centralized blood management systems have limitations in that they lack detailed blood information and, moreover, information is not reflected in real time. To solve this problem, this paper presents an innovative blood cold chain system based on blockchain technology. The proposed system aims to increase information visibility by recording the overall information on the blood supply and providing detailed blood information such as blood consumption and disposal to the distributed ledger. In addition, this paper proposes direct blood transactions between medical institutions in cases of emergency. Currently, blockchain technologies are being actively employed in the supply chain management and medical fields in addition to financial systems. Particularly, private blockchain techniques with limited participants are relatively fast and reliable, making them suitable for B2B (Business-to-Business) transactions. Therefore, the proposed system is based on the architecture of Hyperledger Fabric, a private blockchain technology implemented by the Hyperledger Composer tool. Information in the proposed blood cold chain system cannot be forged or tampered with, and information recorded and shared in real time is kept transparent. In addition, allowing for B2B blood transaction in special circumstances will minimize the blood supply time and enable patients to be transfused quickly. Moreover, the surplus blood of medical institutions will be used to increase the usage rate relative to the supply amount.
\end{abstract}

Keywords: blood cold chain; blockchain; hyperledger; information visibility

\section{Introduction}

The amount of life-saving blood available for transfusion depends on the amount obtained through blood donation. Globally, blood is banned from commercial distribution, and indeed, due to the complexity of blood management, central and/or state governments usually are responsible. In Korea, the Korea Red Cross Blood Management Headquarters was established in 1958. There are 15 blood banks and 3 blood inspection centers related to the blood supply for blood transfusion centers. Domestic blood management is managed by the Blood Information Management System (BIMS) and consists of an inspection system, a supply system, and a mobile system comprising blood donation vehicles.

In the case of medical institutions, the Blood Information Sharing System (BISS) manages donations, blood reservations and orders, test-result inputs and inquiries, as well as blood receipt and delivery. According to transfusion guidelines, medical institutions input blood-receipt and delivery data via 
the Blood Management System (BMS) of the Blood Information Sharing System [1]. Since blood for transfusion is most often consumed or disposed of in medical institutions, information management from a medical institution's point of view is required. Since the blood information of medical institutions is limited to the amounts of blood received and released, determination of the reasons for specific disposals of blood is problematic. According to the World Health Organization (WHO), the blood cold chain represents a system for storing and transporting blood under the appropriate conditions, including temperature, from the blood donor to the final transfusion point [2]. The participants in blood cold chains are blood banks, blood inspection centers, transportation vehicles, and the medical institutions under the blood management headquarters. Due to the uncertainty of blood quality, supply agility among participants is required in order to enable rapid response [3]. Securing information visibility by detecting changes in the supply chain and by sharing information enables a flexible and agile supply chain [4].

Accordingly, once information visibility in the blood cold chain is secured, real-time information sharing and transparent information management among participants can respond to demand efficiently and agilely. Medical institutions secure blood volumes through blood reservations and orders. However, demand uncertainty is high, because the amount of blood required in an emergency that may not occur is constant. The situation is made more difficult because of the inability to use blood after a certain period of time. Research into blood demand response in special situations such as disasters $[3,5,6]$ has been ongoing. However, alternatives, such as changing the location of blood banks, increasing the number of blood banks, and setting up specific medical institutions in the supply area for sudden demand, can lead to cost problems and conflicts of institutional interests [7].

This paper presents a new blood cold chain system design methodology based on private blockchain technology. We describe the proposed architecture, based on which blood transactions between medical institutions can be completed in a fully distributed manner. Due to the functionality of blockchain technology, the proposed blood cold chain system in blockchain can enhance information visibility. Blood banks and blood test centers, transportation vehicles, and medical institutions are participants in the blood cold chain system. Because blood has a large demand uncertainty, supply agility among cold chain participants is required to quickly respond to changes in demand.

Our main contributions are summarized as follows:

- We propose an innovative blockchain-based blood cold chain system in order to overcome the limitations of current centralized blood management systems. Compared with the conventional centralized system, the proposed system can significantly increase the visibility of the information of the blood cold chain system by recording the overall information of blood supply and detailed information such as consumption and disposal in the distributed ledger (see Sections 3.2.1 and 3.2.2).

- We designed a new type of blood supply system that directly transacts blood among medical institutions when a medical institution with an urgent demand cannot receive blood quickly from a blood bank (see Section 3.2.4). In a centralized blood supply system, it is virtually impossible to receive from a supply point other than the blood bank. A fatal problem exists that endangers a patient's life when an emergency occurs in a hospital far from the blood bank.

- We implemented a comprehensive prototype of the blockchain-based blood cold chain system using Hyperledger Composer, of which the implementation demonstrates that information visibility can be gained by creating and tracking a single record per supply point and reducing blood supply time in emergencies (see Section 4).

The rest of the paper is organized as follows: Section 2 reviews the previous research on blood cold chains and the features, types, and techniques of blockchain. It also provides examples of blockchain applications in the supply chain and healthcare sectors. Section 3 presents the new blood cold chain system methodology based on Hyperledger Fabric technology and describes the design method according to two environmental configurations. Section 4 presents the development environment and 
the characteristics of Hyperledger Composer along with its implementation results. Finally, Section 5 concludes the paper.

\section{Related Work}

Research on rapid blood supply and distribution has been conducted by various approaches. This section reviews previous studies on blood cold chain management and various cases of applying blockchain technology to supply chain management are reviewed. In addition, a basic concept of the blood cold chain system using blockchain technology is presented. Finally, the limitations of the existing research and the differentiation of this work are discussed.

\subsection{Blood Cold Chain Management}

Supply chain management integrates core business processes and information through customers and retailers, and wholesalers, manufactures, and suppliers, which adds value for customers and other stakeholders [8]. Supply chain management incorporates very complicated processes that require synchronization of various activities resulting in randomness and supply chain risk $[9,10]$. Lavastre et al. defined supply chain risk management as "the management of risk that implies both strategic and operational horizons for long-term and short-term assessment". Zimon and Madzík [11] determined the impact of standardized management systems on minimizing selected aspects of risk in the supply chain.

Seuring and Müller [12] offered a literature review on sustainable supply chain management and proposed a conceptual framework for the research in this field. They also presented core issues in sustainable supply chain management based on a Delphi study [13]. Saeed and Kersten [14] identified and analyzed drivers of sustainable supply chain management that influence or encourage organizations to endeavor sustainability initiatives and implement sustainable solutions. Zimon et al. [15] characterized the conditions and barriers related to the implementation of United Nation's Sustainable Development Goals (SDGs) in supply chains.

Nagurney et al. [16] proposed a blood supply chain network that minimizes cost and risk by representing the decay characteristics of blood as an arc multiplier. Jabbarzadeh et al. [17] introduced a network design that uses an optimization model for disaster-scenario blood supply. Fahimnia et al. [6] proposed a probabilistic supply chain model that takes into account disaster-scenario cost and delivery time. Armaghan and Pazani [18] designed a blood supply chain to handle urgent requests from blood supply units in the event of an earthquake. They presented a multi-level, multi-objective mathematical model to minimize the cost of the blood supply chain network and maximize the reliability of the selected routes for blood transportation. Eskandari-Khanghahi et al. [19] developed a supply model incorporating mixed-integer linear programming that simultaneously considers location, allocation, inventory, and delivery. Delen et al. [20] sought to support the decision-making process in the blood supply chain using both a geographic information system and data mining techniques.

There are two main problems with the existing blood cold chain that have not been addressed in previous studies. First, there is the problem of insufficient information visibility of the blood information system. Second, there is the problem of delay of blood transportation in an emergency due to a centralized blood supply system. The blood supply chain is not only about storing and distributing blood, but it is also an important issue in the medical field related to a patient's life. Therefore, it is necessary to improve the problem of information sharing in a blood supply chain and to prepare a method to cope with urgent demand by using blockchain technology, as in this research.

\subsection{Application of Blockchain Technology to Supply Chain Management}

Blockchain technology is actively being applied in the financial field because blockchain technology ensures the ease and reliability of tracking financial transactions [21]. Since traceability is also important in supply chain management, it is expected that there will be more cases of blockchain application. Hackius and Petersen [22] conducted an online survey and asked logistics experts for their view on 
use cases, barriers, facilitators, and the future prospects of blockchain in logistics and supply chain management. Walmart adopted a decentralized food supply ecosystem for a food traceability system based on Hyperledger Fabric together with its technology partner IBM [23]. The diamond industry has adopted blockchain technology to achieve greater transparency. De Beers Group first announced a system based on blockchain technology that allows the source and distribution channels of diamonds to be verified through blockchain [24]. This helps consumers to avoid buying fake or stolen diamonds.

Mettler [25] reviewed a variety of application areas of blockchain such as Bitcoins as a starting point, administration and enforcement of music rights, settlement of smart contracts, and health management. The Hyperledger Research Network's Counterfeit Medicines Project uses blockchain to track information such as when and where drugs are produced in order to prevent the production of counterfeit drugs [26]. Drugs are marked with a timestamp, and theft and forgery can be identified, increasing the safety of the drug. These examples show that blockchain technology can be used for supply chain management to enable secure purchases and facilitate tracking when supply channels are complex.

As for the cases of blockchain technology application in the medical field, Kim [27] implemented a medical examination result repository using Hyperledger Fabric technology to secure the accessibility of patients' personal information and to manage security, which is essential for medical information. Yang [28] applied blockchain to a medical system for improved security of identity authentication and easy access of EMR (Electronic Medical Record) information using smart contract features. The application of blockchain technology in the medical field is expanding, especially in the U.S.A and various private companies, for the purposes of storing and exchanging medical records [29].

\subsection{Hyperledger Fabric}

The blockchain technology that emerged with Bitcoin is a decentralized, distributed database technology. Data from system databases can be shared over a network, allowing for real-time sharing even in a distributed environment, and maintaining consistent data by means of a consensus algorithm [30]. Blockchain, also called distributed ledger technology, consists of a number of blocks, which contain information on a number of transactions. Participants (nodes) of the blockchain network can make direct transactions without a relay or a third party, and since the block is verified, it adds only approved blocks, thereby ensuring reliability. In addition, since the blocks are arranged by time, it is difficult to manipulate the contents of the preceding block because all of the following blocks need to be regenerated. The blockchain system consists of several technologies, including peer-to-peer networks, consensus algorithms, digital signatures and hashes, and smart contracts [28,31].

Depending on the characteristics of the blockchain network, it can be classified into public, private, and consortium [30,32]. Public blockchain networks have no restrictions on participants, allowing them to create, verify, and approve transactions. However, all nodes participating in the network have the same authority and are slowed down by multiple participants performing transaction approval. Private blockchain networks, on the other hand, only authorize users who can participate. The consortium blockchain is semicentral in that it has the form of a network in which several authorized institutions participate, not just one, but it has the characteristics of a private blockchain. Typical blockchain-based technologies include Bitcoin Core, Ethereum, and Hyperledger Fabric. Bitcoin Core is a representative public blockchain network whereby transaction records are shared among all participants and written in blocks every $10 \mathrm{~min}$. Transactions are executed in a scripting language, for which the scheme is very simple and has the disadvantage of lacking extensibility. Another public blockchain-based technology, Ethereum, is a platform that can execute smart contracts on a blockchain basis and records not only transaction history but also smart contracts and their execution history.

Hyperledger Fabric is a Linux Foundation project that was launched with 17 member companies in December 2015. The alpha version of the software was released on March 2017, and Hyperledger Fabric 1.0 was released in June. Figure 1 shows Hyperledger Fabric's private network and ledger. The most distinctive feature of Bitcoin's block is the Key-Value Store (KVS). KVS preserves transaction processing results. Blocks store transaction information and hash values of the KVS. 


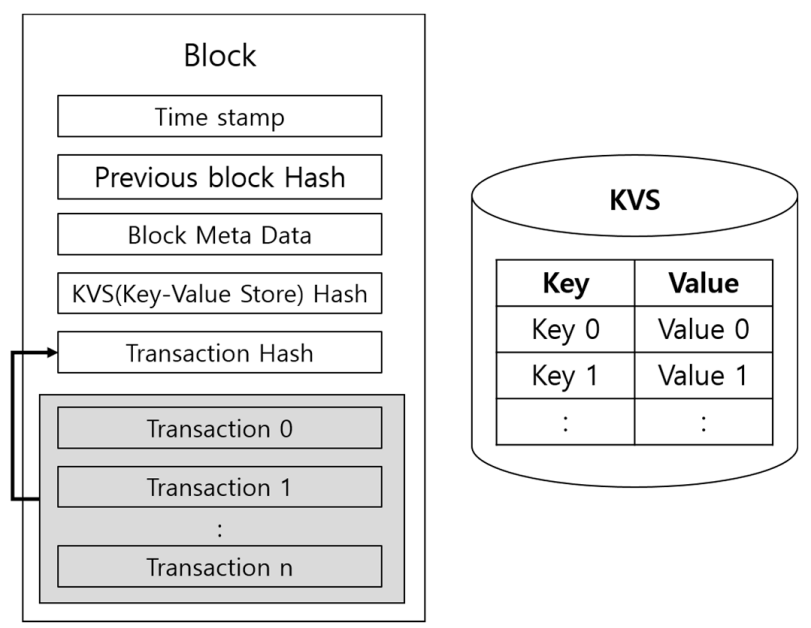

Figure 1. Block of Hyperledger Fabric and KVS.

Hyperledger Fabric provides three representative services: a membership service, a consensus algorithm, and a chaincode service [32]. First, the membership service manages the participants' information in order to give the blockchain network limited privileges. Second, Hyperledger Fabric takes a different approach to consistency from that of Bitcoin Core or Ethereum. A consensus algorithm called PBFT (Practical Byzantine Fault Tolerance) is used to obtain the consensus of participants when updating data. In the PBFT method, a specific node acts as a leader, sends a request to all nodes including itself, aggregates the response, and then confirms the block. Third, chaincode is a program that handles transaction execution. It implements processing such as init, invoke, and query. Unlike the previous 0.6 version, the biggest change in version 1.0 is the role of peers. Validating peer, which plays a major role in consensus algorithms and ledger management, is divided into endorsing peer, committer peer, and ordering node in Version 1.0.

Figure 2 shows the architecture of Hyperledger Fabric 1.0. Endorsing peer performs the task of verifying the transaction proposal, and the ordering service sequentially arranges for each peer to store a consistent ledger for the transaction; committer peer performs the task of storing the ledger through transaction verification.

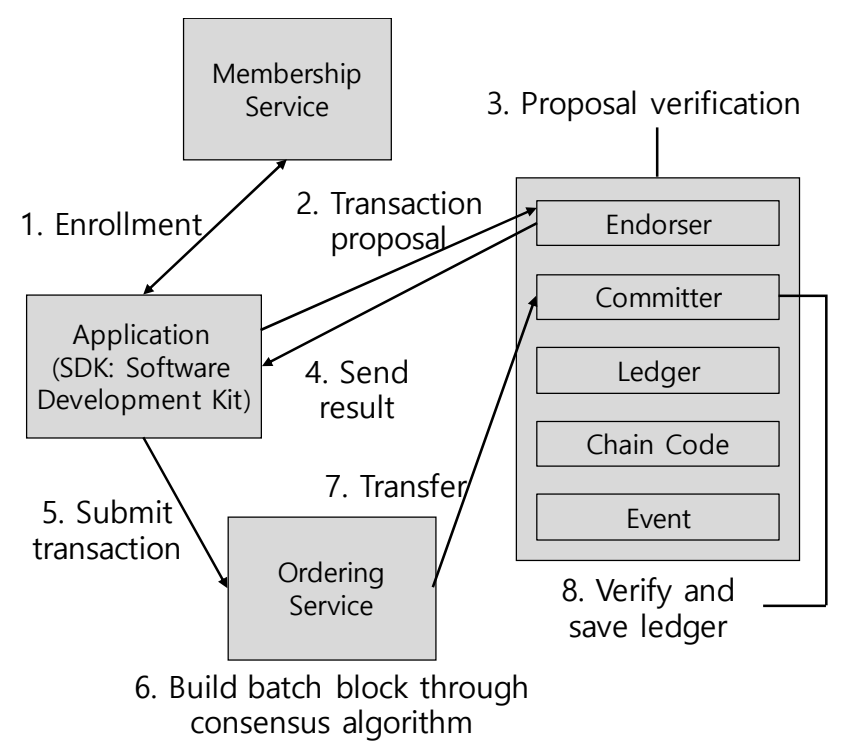

Figure 2. Architecture of Hyperledger Fabric 1.0. 


\subsection{Limitations of the Existing Research}

Currently, the blood cold chain system has problems with supply time and information management due to its centralized structure. Most of the studies conducted to solve the supply time problem have focused on mathematical models with objective functions and determine the number, location, or delivery route of blood sources. However, adjustment of the blood supply region may cause conflicts of interest between blood sources, avoidance of roles of medical institutions, accountability, and cost issues.

This study aims to design and implement a new blood cold chain system based on blockchain technology to improve the above problems. Blockchain technology makes it difficult to forge and falsify information, allowing the system to be managed transparently in real time. In addition, blockchain-based smart contracts can save time and effort related to document processing, and have a feature of speeding up execution. To solve the supply time problem by using these advantages, blood transactions between hospitals are designed and executed as smart contracts in emergency situations. In addition, in the case of disposing of blood in the hospital, which is the final consumption place of donated blood, a method in which the cause can be recorded in real time can be provided. Therefore, the proposed system overcomes the limitations of the existing system and prior research by considering both the aspects of the existing blood information system and the supply time.

\section{Design of Blood Cold Chain System}

\subsection{Overview}

In this paper, we propose a blood cold chain system design using Hyperledger Fabric, a private blockchain technology. We discuss design scenarios and architectures, specifically for emergency blood requests. The most important thing for hospitals that suddenly need blood is a ready blood supply. However, if the blood bank that supplies the blood is far from the hospital, difficulties of supply occur. These difficulties can be solved by using surplus blood from other hospitals that are closer. Therefore, we designed a system for direct, emergency-scenario blood exchange between nearby hospitals. Using blockchain technology, the relevant transaction records are recorded and shared in real time.

\subsection{New Blood Cold Chain System Based on Blockchain Technology}

\subsubsection{Scenarios of New Blood Cold Chain System}

The system is divided into two scenarios. Both concern situations in which donated blood is used for transfusion. The general situation is that the blood bank of the Korea Red Cross Blood Management Headquarters produces blood for transfusion, sends blood to the test center for safety, and then supplies the blood at the request of hospitals. Under general conditions, hospitals assume that they cannot order blood from a blood bank other than the designated one.

On the other hand, in the case of an emergency, the blood supply request is made according to the new method proposed in this paper. If a specific hospital needs a blood supply within 60 min due to a lack of stock, blood is ordered and received from nearby hospitals if certain conditions are met. In this case, the consensus process prevents false requests in advance and maintains the system so that only transactions that meet the conditions can occur.

\subsubsection{Architecture of New Blood Cold Chain System}

The blood cold chain consists of the blood management headquarters, blood banks, blood inspection centers, transportation vehicles, and hospitals. Because the participants are specified, the private blockchain approach is appropriate. The participants register using the membership service of Hyperledger Fabric. Figure 3 shows the existing blood cold chain and the architecture of the proposed new system. The left side of the figure shows the conventional centralized form, and the right side depicts the proposed blood cold chain system with the shared distributed branching technology. 
By this blockchain technology, the network participants share the same distributed ledger, and the blood management headquarters plays the role of information inquiry and transaction monitoring rather than participating directly in the supply chain.

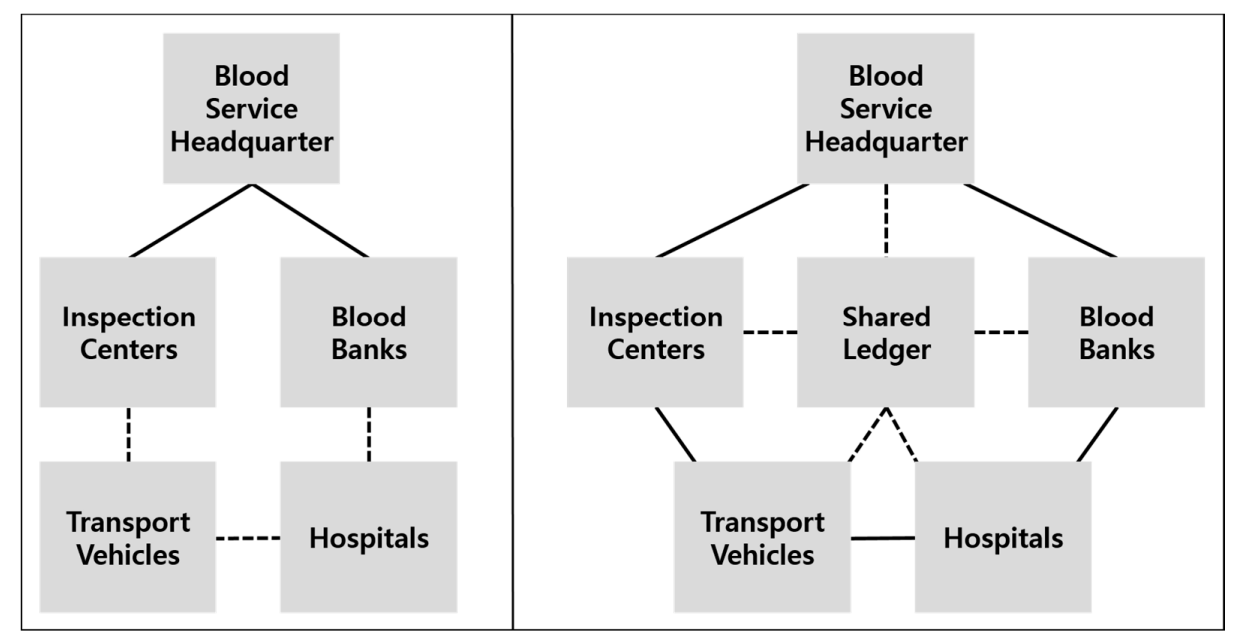

Figure 3. Conventional centralized blood cold chain system (left); proposed blood cold chain system (right).

\subsubsection{General Blood Supply Situation}

The distributed ledger architecture of the blockchain blood cold chain system proposed in this paper is shown in Figure 4. It is based on the block structure of Hyperledger Fabric, and shows the transactions that are created each time blood flows through its base.

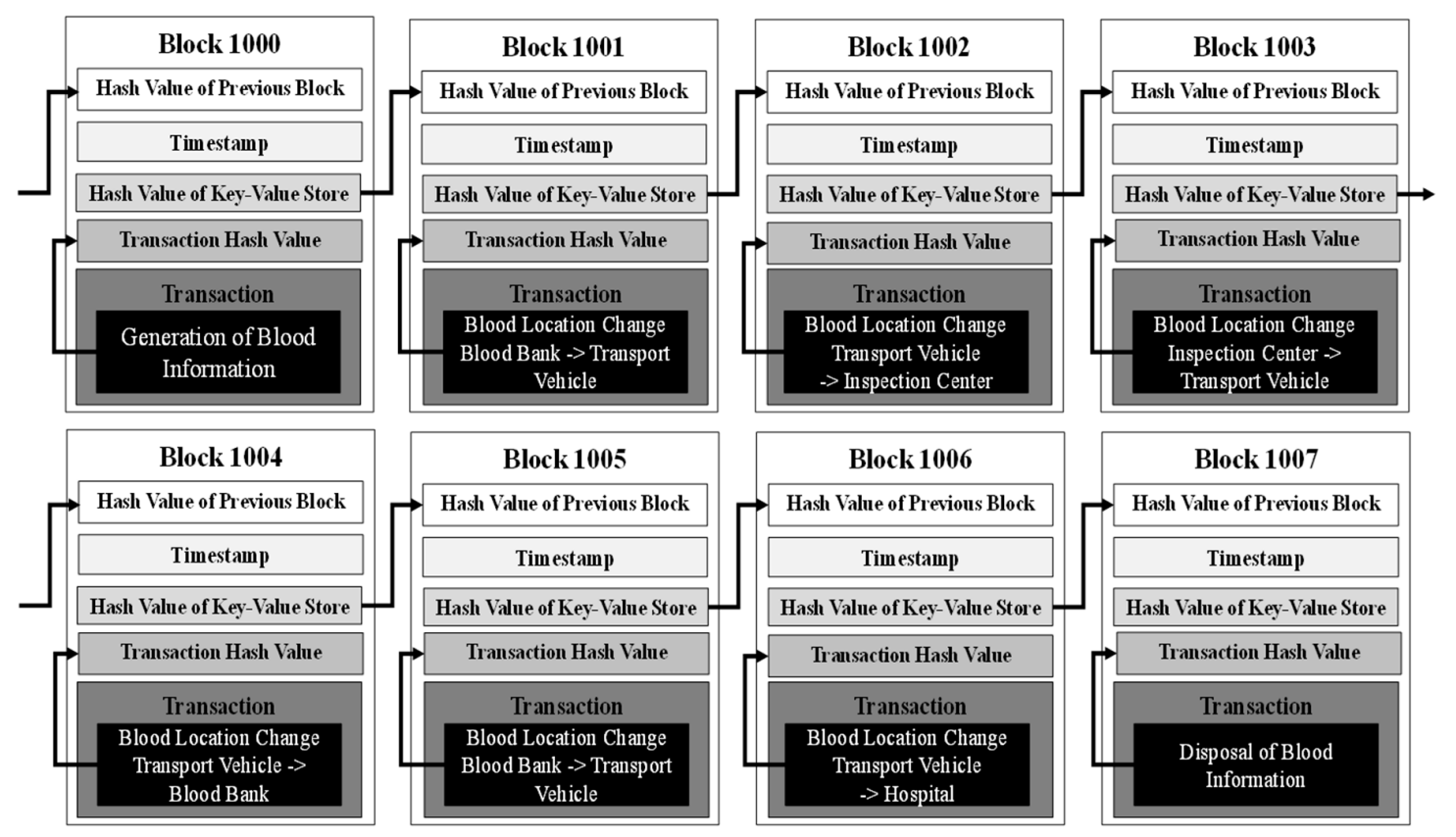

Figure 4. Architecture of distributed ledger.

There are transactions in Block 1000, where 15 blood banks across the country produce blood for transfusions, and in Block 1001 and Block 1002, there are transactions from a blood bank to an inspection center via transportation vehicles. Transactions that send blood back to the blood bank can be confirmed by Block 1003 and Block 1004. Block 1005 and Block 1006 represent the transfer of blood from a blood bank to a hospital in the event of a blood order. Finally, Block 1007 shows the use of blood 
stored in a hospital. Each block's KVS is responsible for preserving transaction processing results to ensure an up-to-date status. The KVS has the same contents in all verification codes, and the hash value is recorded in the blockchain.

As blood is a temperature-sensitive resource requiring the use of a dedicated refrigerator to maintain a constant temperature, the new system checks the temperature and records the transaction to check the temperature. This is done to ensure that blood having a temperature management problem is disposed of. In Hyperledger Fabric, chaincode is a program for executing transactions, which is the basis of smart contract execution. Therefore, a transaction in Hyperledger Fabric can be interpreted as the execution of chaincode. When checking temperature information at a specific point in time, it can be recorded as a transaction through chaincode, which is recorded in the blood asset information.

One of the important reasons for implementing the proposed blood cold chain system in blockchain is information visibility. Many medical institutions wherein blood transfusions are made generate data that records when the final blood is used or disposed of, which can improve blood inventorying as well as blood-demand predictability. The participant consensus algorithm of Hyperledger Fabric sequentially records transactions over time and manages information transparently so as to prevent forgery or alteration.

\subsubsection{Emergency Blood Supply Situation}

In an emergency, a medical institution far from the blood bank will not be able to receive a blood supply quickly. To address the limitations of the existing centralized blood supply, the system designates this as a special situation and allows the medical institution to request the type of blood and the amount of blood required from nearby medical institutions. Figure 5 shows the transaction flow and consensus process for this situation. Since Hyperledger Fabric uses a distributed consensus algorithm via a specific verification node, the role of verifying the requested transaction in this case is the peer of hospital A and the peer of hospital A's neighbor (hospital B).

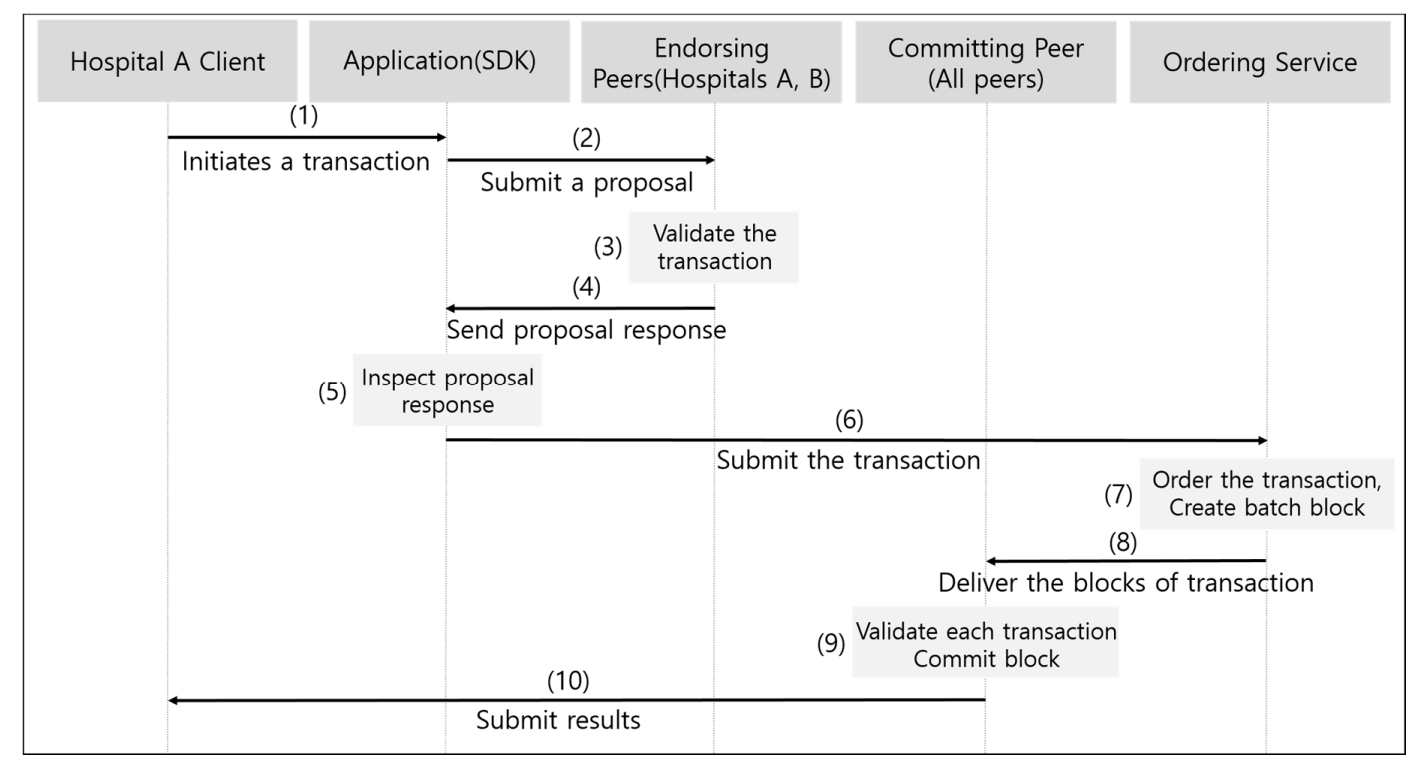

Figure 5. Blood supply in emergency situations.

(1) shows that the client of hospital A requests a blood transaction. (2) and (3) show that when the proposal is delivered to the assurance peer, the peers of hospital A and the neighboring hospital (hospital B) corresponding to the assurance peer execute the chaincode to verify the transaction. In (4) and (5), the application examines the results received from the guaranteed peer. (6) shows that the application submits the transaction to the ordering service. In (7) and (8), the ordering service defines the transaction sequence, creates one block, and sends it to all peers. (9) shows that each peer verifies the 
transaction and confirms the transaction after verifying that the guarantee condition is met. (10) shows that the neighboring hospital (hospital B) ultimately supplies the surplus blood supply to hospital A.

\section{Implementation of the New Blood Cold Chain System}

\subsection{Hyperledger Composer}

The proposed system is implemented using Hyperledger Composer, an open-source application development tool based on Hyperledger Fabric technology [33,34]. This tool, based on JavaScript, uses the blockchain business network, because it is suitable for a private blockchain business environment.

\subsection{Implementation in General Situations}

The participants in the blood cold chain system network are defined in the model file. One is the participant registry list and another is the asset registry list. Both lists are composed of ID and data. The participant types in this network are blood banks, inspection centers, transportation vehicles, and hospitals. To add more participants, the ID and data are defined using "create new participant".

As noted earlier, blood is a temperature-sensitive resource, and so temperature information must be obtainable in real time. In the proposed system, therefore, we implemented a temperature verification transaction as shown in Figure 6.

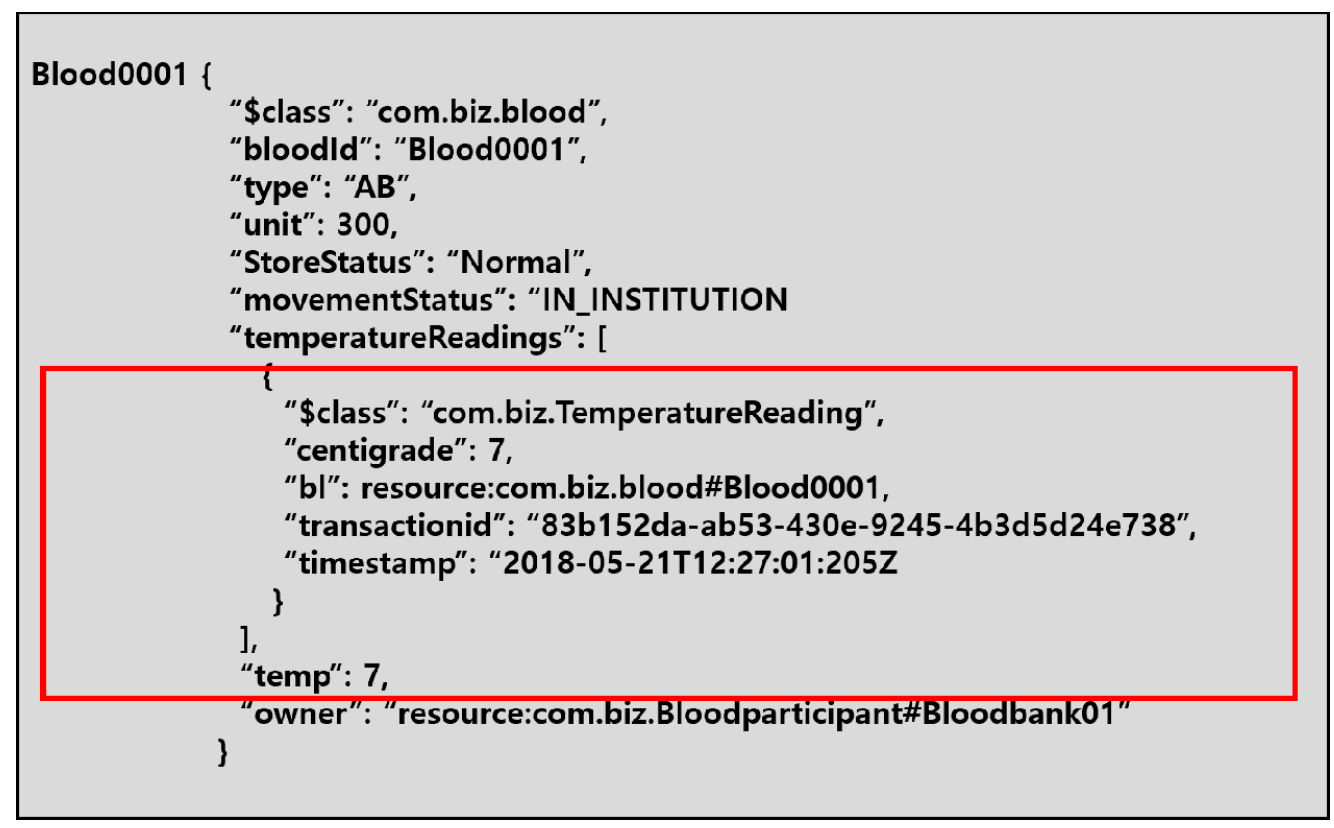

Figure 6. After running of temperature-check transaction.

Initial temperature information is optional. Then, when a temperature verification transaction occurs, a time stamp is recorded to indicate when the temperature was confirmed. Collected blood should be stored at 1 to 6 degrees Celsius, which means that any blood outside the storage reference temperature should be discarded after a temperature verification transaction. Discarding of blood requires a separate transaction and function. A transaction is specified in the model file, and a script file is written to apply the function to the transaction.

Scripts in Hyperledger Composer are useful for executing developer-defined code or specific commands, and can be interpreted as spaces that define some sort of transaction execution function. This corresponds to the business logic of the blockchain network and is written in JavaScript (.js). In addition, it uses the Query Language supported by Hyperledger Composer to find specific blood that is outside of the standard temperature range. When the network is configured, a separate query 
file (.qry) is created. In this case, the conditions are set using the SELECT and WHERE operators. Events can be specified separately for details after a transaction to discard blood has taken place.

In the left of Figure 7, the blood information shows that the current temperature is outside the whole blood storage temperature range of 0 degrees Celsius to 8 degrees Celsius. Event details can be specified separately after a transaction to discard blood has taken place. This is specified in the model file along with the transaction. Detailed logic can be specified through a function in the script file. As shown in the right of Figure 7, there are three events that occurred at the time the transaction was executed. This information can be used to determine which blood was discarded and when. The event shows that Blood0007 and Blood0008, blood outside of the storage reference temperature shown in the left of Figure 7, were discarded.
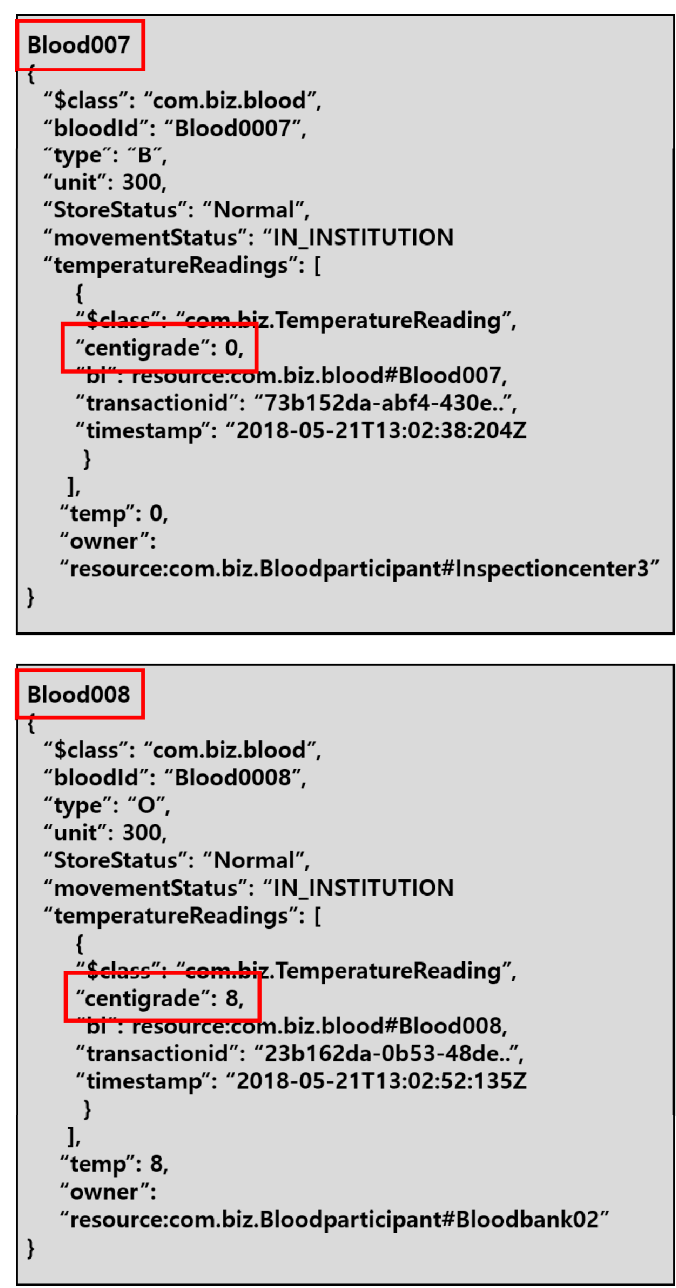

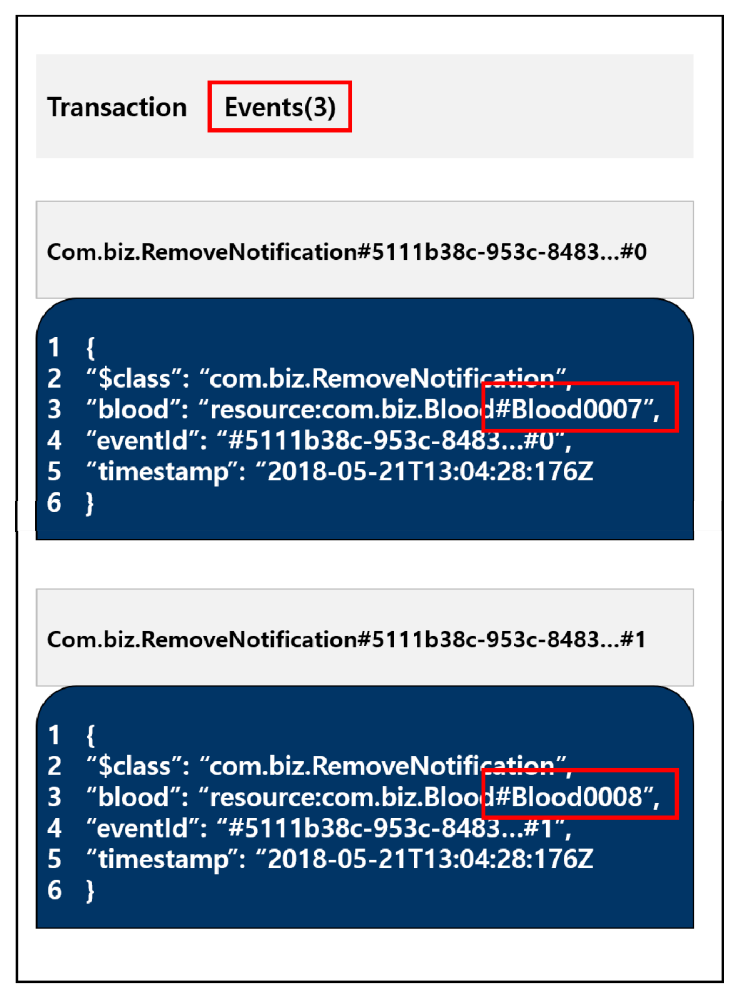

Figure 7. Events for blood discard transaction by temperature condition.

Additionally, blood may be discarded for reasons such as turbidity and discoloration. To manage this, we defined properties that indicate the state of storage in the blood information. This attribute is required and should be entered when all of the initial blood assets are created. In addition, participants in the blood cold chain update the cause when the current blood storage status changes. At this time, the blood whose revised storage condition is not normal is removed through a blood disposal transaction by the storage condition. A query was defined as shown in Figure 8 to search for blood whose status is to be "discarded". 


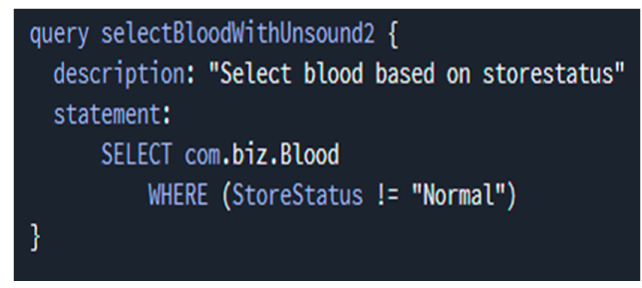

Figure 8. Query to search for blood subject to disposal.

Blood is characterized by a specific shelf life depending on its ingredient formulation. Therefore, in addition to the temperature and storage conditions, it is necessary to continuously check the storage period and dispose of it after that period. After one day in the system, the retention period of the entire blood asset is reduced by one day. As with the temperature and storage conditions, we designed a transaction that discards blood when the retention period reaches zero days. It also allows the user to view the quantity of blood assets that have a retention period of about one day. The blood moves according to the blood supply process for transfusion.

The movement state is defined to indicate whether the blood is currently moving or within the organ. In addition, the blood ownership information is defined, and the first blood owner is the blood bank. In Figure 9, the first part represents the first blood, indicating that the movement is within the organ and that the owner is the blood bank. The second part shows that when the blood starts to go to the inspection center, it changes to the status of "moving". The third part shows that after the blood is moved to the inspection center, the owner has been changed from the blood bank to the inspection center. When a transaction is performed to check the blood temperature within the inspection center, the results are recorded in the blood asset details. The fourth part shows that after the blood is judged to be suitable for donation, it moves again to the blood bank.
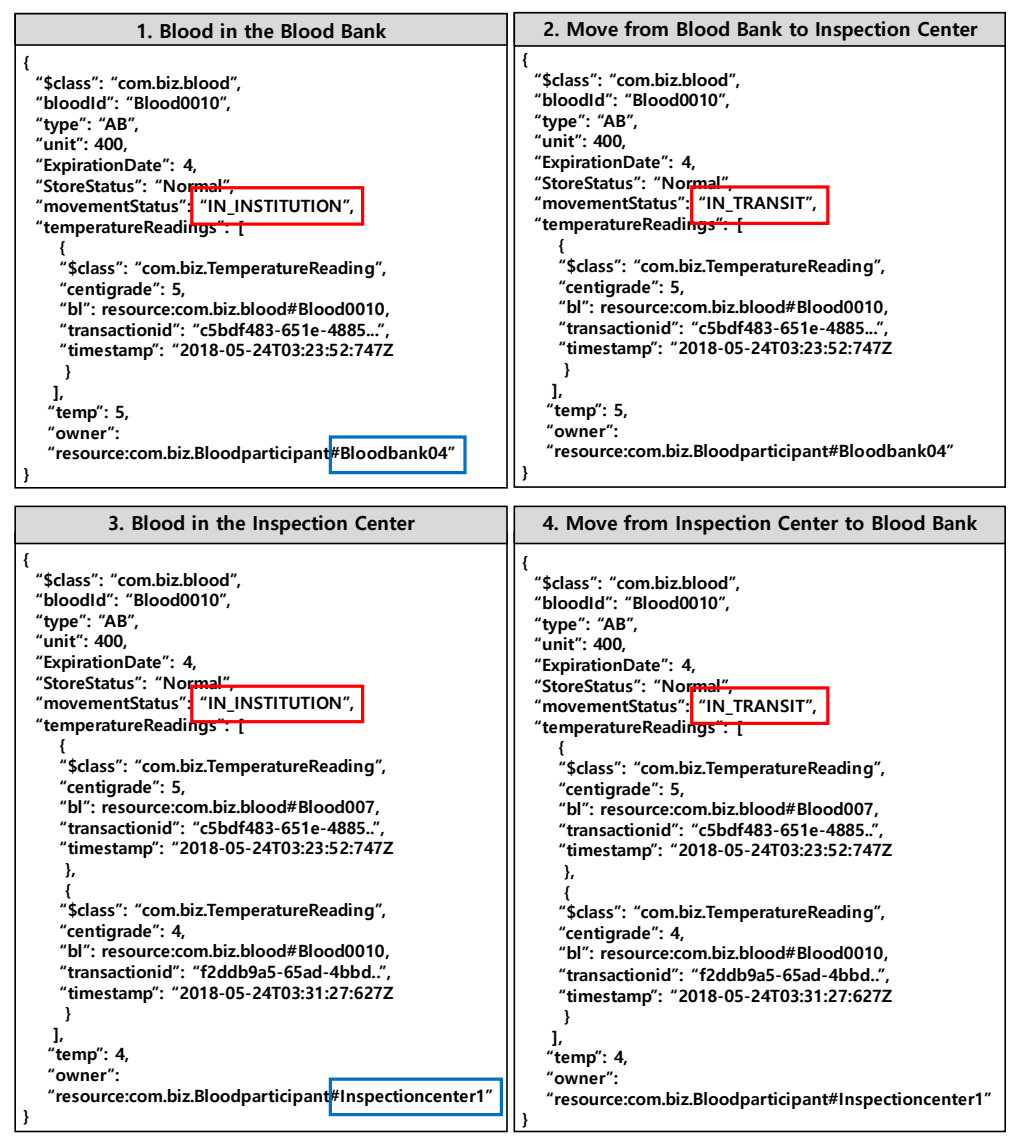

Figure 9. Blood movement in general situations. 


\subsection{Implementation in Emergency Situations}

In emergencies, hospitals that are far from the blood bank have longer supply times. Thus, we defined the business logic in this system to allow blood to be exchanged between hospitals in such situations.

The logic can be divided into two types. First, the requesting hospital must have an emergency condition. Second, the requesting hospital must have surplus blood and supply blood to other hospitals. Whether or not an emergency occurs, supply availability is defined as a property of the participant registry, and the hospital can modify this information at any time. If the two logics do not hold at the same time, the system informs the user that a transaction cannot be made.

In Figure 10, the first part represents blood (Blood0013) inside hospital 1 (Hospital0045). The second part show that the flow of blood changes to requests allowed (REQUEST_ACCEPT). The fourth part show that when the inter-hospital blood request transaction is complete, the owner of the blood is changed to hospital 2 (Hospital0046), where the emergency occurred.

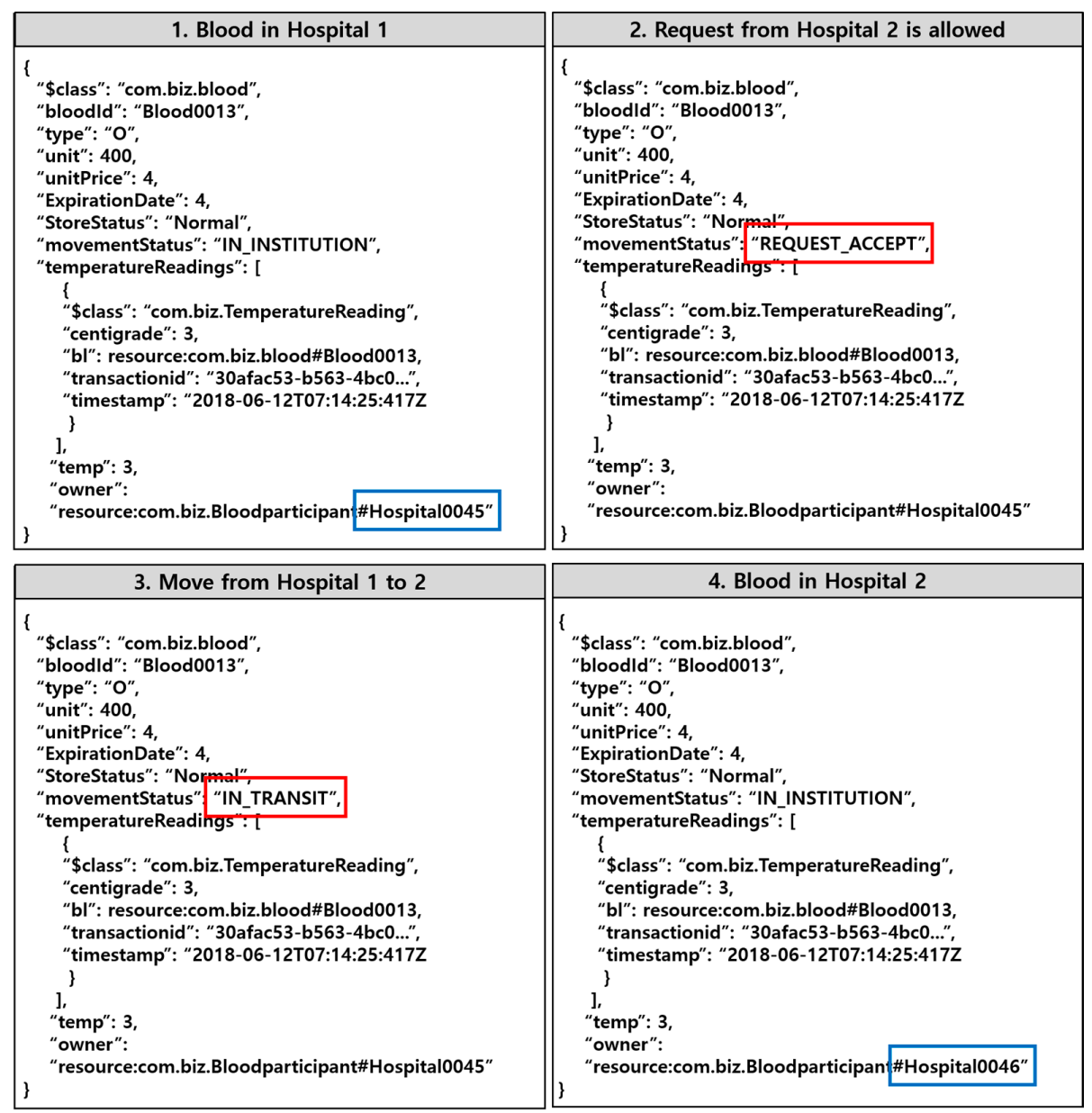

Figure 10. Blood supply in emergency situations.

In the proposed system, we have an environmental scheme in which payment transactions occur when blood requests occur between hospitals. Since this is not an order form from an existing blood bank, payment is made in the form of blood requests between hospitals. Figure 11 shows that the asset data is changed by the transaction between hospital 1 and hospital 2. The trading amount is the blood unit price multiplied by the trading volume. The blood unit price of the trading target is assumed to be 4 and the trading volume is 400 . Accordingly, the total transaction amount is 1600 . In addition, the pre-transaction assets of hospital 1 and hospital 2 were set at 7000 and 10,000. Therefore, after the 
transaction, hospital 1 receives 1600 from hospital 2 and has 8600, and hospital 2 has 8400, as seen in the figure below.

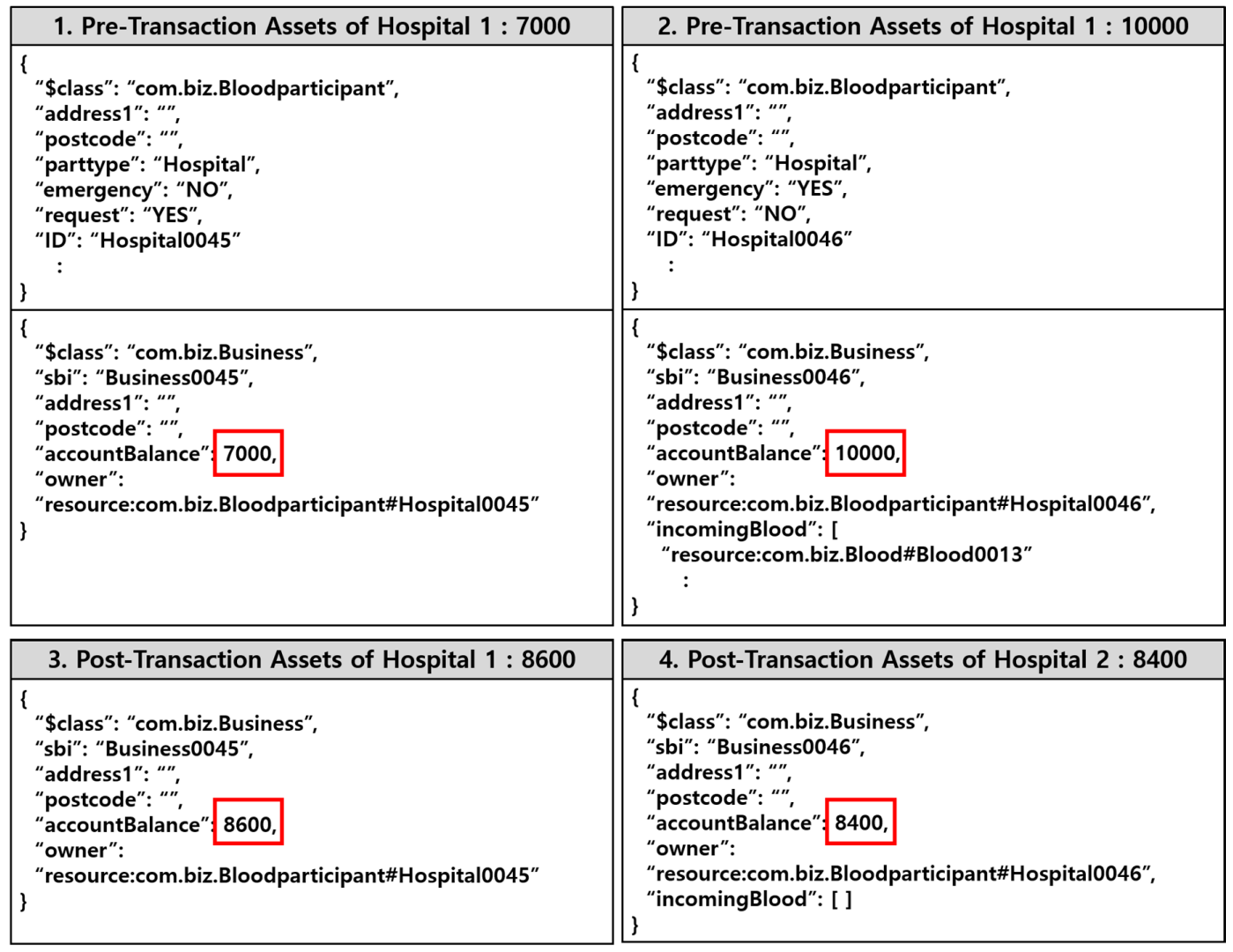

Figure 11. Hospital assets before and after executing blood request transaction.

\section{Conclusions}

We designed and implemented a blood cold chain system based on the private blockchain technology to achieve two goals: secure information visibility and reduce blood supply time. First, the real-time recording and sharing of information as blood is moved, consumed, and discarded in distributed ledgers enables efficient management of blood. In addition, this system, which prevents forgery and information tampering, will make the blood management operation more transparent by solving problems that may occur in medical institutions and with medical staff, such as missing inputs of entry, exit, and errors. Second, the system, which supports blood transactions between medical institutions after consensus is reached on urgent demand, has the significance of reducing the blood supply time, which is directly related to saving the lives of emergency patients. This also means that hospitals can use their surplus blood efficiently, because they can keep their blood stock intact while maintaining excess blood inventory.

Currently, the use of blockchain technology in the medical field is actively discussed, but there are no previous relevant studies or application examples in the blood cold chain field. For realization of the blockchain network proposed by this paper, consideration should be given to coordination of interests among participants in the blood cold chain system as well as the infrastructural, legal, institutional, and technical aspects.

Although this study proposes an innovative method for blood cold chain management and implementation of a prototype system, there is insufficient verification of the proposed system. Therefore, in the future, we will perform a thorough evaluation study in order to prove the effectiveness of the proposed scheme by integrating the proposed system with the actual blood information system. Furthermore, we will consider the proposed design's cost and network configuration and consider 
the additional information, block size, and hacking-prevention potential required for actual hospital blood transactions.

Author Contributions: Conceptualization, S.K. and D.K.; methodology, S.K.; software, S.K.; supervision, D.K.; writing - original draft, S.K.; writing —review and editing, J.K. and D.K. All authors have read and agreed to the published version of the manuscript.

Funding: This work was supported by Basic Science Research Program through the National Research Foundation of Korea (NRF) funded by the Ministry of Education (NRF-2017R1D1A1B05029080).

Conflicts of Interest: The authors declare no conflict of interest.

\section{References}

1. Chung, G.S. Transfusion Guidelines, 4th ed.; Korea Centers for Disease Control and Prevention: Wusongyi, Korea; Cheongju, Korea, 2016.

2. World Health Organization. The Blood Cold Chain. Available online: http://www.who.int/bloodsafety/proc essing/who_eht_11_04_en.pdf?ua=1 (accessed on 10 April 2020).

3. Hess, J.R.; Thomas, M.J.G. Blood use in war and disaster: Lessons from the past century. Transfusion 2003, 43, 1622-1633. [CrossRef] [PubMed]

4. McCrea, B. EMS Completes the Visibility Picture. Logist. Manag. 2005, 44, 57-61.

5. Chung, B.D.; Kim, S.I. Agile Blood Supply Chain Design Considering Golden Time. Korean J. Logist. 2016, 24, 21-41.

6. Fahimnia, B.; Jabbarzadeh, A.; Ghavamifar, A.; Bell, M. Supply chain design for efficient and effective blood supply in disasters. Int. J. Prod. Econ. 2017, 183, 700-709. [CrossRef]

7. Kim, C.B.; Park, S.A. A Study on the factor of Supply Chain Management Success on Blood Supply Chain. J. Internet Electron. Commer. Res. 2008, 8, 237-259.

8. Kim, D. An Integrated Supply Chain Management System: A Case Study in Healthcare Sector. Lect. Notes Comput. Sci. 2005, 3590, 218-227.

9. Shahbaz, M.S.; Rasi, R.Z.R.M.; Ahmad, M.F.B.; Rehman, F. What is Supply Chain Risk Management? A Review. J. Comput. Theor. Nanosci. 2019, 23, 9233-9238. [CrossRef]

10. Lavastre, O.; Gunasekaran, A.; Spalanzani, A. Effect of firm characteristics, supplier relationships and techniques used on Supply Chain Risk Management (SCRM): An empirical investigation on French industrial firms. Int. J. Prod. Res. 2014, 52, 3381-3403. [CrossRef]

11. Zimon, D.; Madzík, P. Standardized management systems and risk management in the supply chain. Int. J. Qual. Reliab. Manag. 2019, 305-327. [CrossRef]

12. Seuring, S.; Müller, M. From a literature review to a conceptual framework for sustainable supply chain management. J. Clean. Prod. 2008, 16, 1699-1710. [CrossRef]

13. Seuring, S.; Müller, M. Core Issues in Sustainable Supply Chain Management-A Delphi Study. Business Strategy Environ. 2008, 17, 455-466. [CrossRef]

14. Saeed, M.A.; Kersten, W. Drivers of Sustainable Supply Chain Management: Identification and Classification. Sustainability 2019, 11, 1137. [CrossRef]

15. Zimon, D.; Tyan, J.; Sroufe, R. Drivers of sustainable supply chain management: practices to alignment with un sustainable development goals. Int. J. Qual. Res. 2019, 14, 219-236. [CrossRef]

16. Nagurney, A.; Masoumi, A.H.; Yu, M. Supply chain network operations management of a blood banking system with cost and risk minimization. Comput. Manag. Sci. 2012, 9, 205-231. [CrossRef]

17. Jabbarzadeh, A.; Fahimnia, B.; Seuring, S. Dynamic supply chain network design for the supply of blood in disasters: A robust model with real world application. Transp. Res. E Logist. Transp. Rev. 2014, 70, 225-244. [CrossRef]

18. Armaghan, N.; Pazani, N.Y. A Model For Designing A Blood Supply Chain Network To Earthquake Disasters (Case Study: Tehran City). Int. J. Qual. Res. 2019, 13, 605-624. [CrossRef]

19. Eskandari-Khanghahi, M.; Tavakkoli-Moghaddam, R.; Taleizadeh, A.A.; Amin, S.H. Designing and optimizing a sustainable supply chain network for a blood platelet bank under uncertainty. Eng. Appl. Artif. Intell. 2018, 71, 236-250. [CrossRef] 
20. Delen, D.; Madhav, M.; Mayer, R.J.; Wu, C.N. Better management of blood supply-chain with GIS-based analytics. Ann. Oper. Res. 2011, 185, 181-193. [CrossRef]

21. Kim, E. A Study for the Innovativeness of Blockchain. J. Soc. E Bus. Stud. 2018, 23, 173-187. [CrossRef]

22. Hackius, N.; Petersen, M. Blockchain in logistics and supply chain: Trick or treat? In Proceedings of the Hamburg International Conference of Logistics (HICL), Hamburg, Germany, 12-14 October 2017.

23. Hyperledger Case Study. How Walmart Brought Unprecedented Transparency to the Food Supply Chain with Hyperledger Fabric. Available online: https://www.hyperledger.org/resources/publications/walmart-c ase-study (accessed on 6 May 2020).

24. De Beers Group. De Beers Group Progresses Development of First Blockchain Initiative to Span the Diamond Value Chain. Available online: https://www.debeersgroup.com/media/company-news/2018/de-beers-groupprogresses-development-of-first-blockchain (accessed on 6 May 2020).

25. Mettler, M. Blockchain technology in healthcare: The revolution starts here. In Proceedings of the 2016 IEEE 18th International Conference on e-Health Networking, Applications and Services (Healthcom), Munich, Germany, 14-16 September 2016.

26. Taylor, P. Applying Blockchain Technology to Medicine Traceability. Available online: https://www.securingindust ry.com/pharmaceuticals/applying-blockchain-technology-to-medicine-traceability/s40/a2766/\#.V5mxL_mLTIV (accessed on 6 May 2020).

27. Kim, M. Blockchain Based Health Checkup Results CDA Viewing Service; Kyungpook National University: Daegu, Korea, 2017.

28. Yang, S. Proposal for Smart Contract Method for Domestic Medical System Based on the Colored Coin; Soonchunhyang University: Asan, Korea, 2017.

29. Choi, H. Use of Block-Chain Technology in the Healthcare Industry; Korea Health Industry Development Institution: Cheongju, Korea, 2017.

30. Crosby, M.; Pattanayak, P.; Verma, S.; Kalyanaraman, V. Blockchain technology: Beyond bitcoin. Appl. Innov. 2016, 2, 6-10.

31. Merkle, R.C. A digital signature based on a conventional encryption function. In Proceedings of the a Conference on the Theory and Applications of Cryptographic Techniques, Santa Barbara, CA, USA, 16-20 August 1987.

32. Introduction to Hyperledger Business Blockchain Design Philosophy and Consensus. Available online: https://ww w.hyperledger.org/wp-content/uploads/2018/07/HL_Whitepaper_IntroductiontoHyperledger.pdf (accessed on 10 April 2020).

33. Hyperledger Composer. Available online: https://hyperledger.github.io/composer/latest/ (accessed on 10 April 2020).

34. Hyperledger Composer Overview. Available online: https://www.hyperledger.org/projects/composer (accessed on 10 April 2020). 